University of New Hampshire

University of New Hampshire Scholars' Repository

Physics Scholarship

Physics

8-2014

\title{
Deep dielectric charging of regolith within the Moon's permanently shadowed regions
}

Andrew P. Jordan

University of New Hampshire, A.P.Jordan@unh.edu

T. J. Stubbs

Goddard Space Flight Center

Jody K. Wilson

University of New Hampshire, jody.wilson@unh.edu

Nathan A. Schwadron

University of New Hampshire, Nathan.Schwadron@unh.edu

Harlan E. Spence

University of New Hampshire, harlan.spence@unh.edu

See next page for additional authors

Follow this and additional works at: https://scholars.unh.edu/physics_facpub

Part of the Physics Commons

\section{Recommended Citation}

Jordan, A.P., Stubbs, T.J., Wilson, J.K., Schwadron, N.A., Spence, H.E., Joyce, C.J. Deep dielectric charging of regolith within the Moon's permanently shadowed regions. (2014) Journal of Geophysical Research E: Planets, 119 (8), pp. 1806-1821. doi:10.1002/2014JE004648

This Article is brought to you for free and open access by the Physics at University of New Hampshire Scholars' Repository. It has been accepted for inclusion in Physics Scholarship by an authorized administrator of University of New Hampshire Scholars' Repository. For more information, please contact Scholarly.Communication@unh.edu. 
Authors

Andrew P. Jordan, T. J. Stubbs, Jody K. Wilson, Nathan A. Schwadron, Harlan E. Spence, and Colin J. Joyce 


\section{Journal of Geophysical Research: Planets}

\section{RESEARCH ARTICLE \\ 10.1002/2014JE004648 \\ Deep dielectric charging of regolith within the Moon's permanently shadowed regions}

Key Points:

- Energetic charged particles deep dielectrically charge the lunar regolith

-We model the resulting subsurface electric fields

- The electric fields may be great enough to induce dielectric breakdown

Correspondence to: A. P. Jordan,

a.p.jordan@unh.edu

\section{Citation:}

Jordan, A. P., T. J. Stubbs, J. K. Wilson, N. A. Schwadron, H. E. Spence, and C. J. Joyce (2014), Deep dielectric charging of regolith within the Moon's permanently shadowed regions, $J$. Geophys. Res. Planets, 119, 1806-1821, doi:10.1002/2014JE004648.

Received 11 APR 2014

Accepted 2 JUL 2014

Accepted article online 4 JUL 2014

Published online 8 AUG 2014

\section{Introduction}

\author{
A. P. Jordan ${ }^{1}$, T. J. Stubbs ${ }^{2}$, J. K. Wilson ${ }^{1}$, N. A. Schwadron ${ }^{1}$, H. E. Spence ${ }^{1}$, and C. J. Joyce ${ }^{1}$ \\ ${ }^{1}$ Institute for the Study of Earth, Oceans, and Space, University of New Hampshire, Durham, New Hampshire, USA, ${ }^{2}$ NASA \\ Goddard Space Flight Center, Greenbelt, Maryland, USA
}

Abstract Energetic charged particles, such as galactic cosmic rays (GCRs) and solar energetic particles (SEPs), can penetrate deep within the lunar surface, resulting in deep dielectric charging. This charging process depends on the GCR and SEP currents, as well as on the regolith's electrical conductivity and permittivity. In permanently shadowed regions (PSRs) near the lunar poles, the discharging timescales are on the order of a lunation ( 20 days). We present the first predictions for deep dielectric charging of lunar regolith. To estimate the resulting subsurface electric fields, we develop a data-driven, one-dimensional, time-dependent model. For model inputs, we use GCR data from the Cosmic Ray Telescope for the Effects of Radiation on board the Lunar Reconnaissance Orbiter and SEP data from the Electron, Proton, and Alpha Monitor on the Advanced Composition Explorer. We find that during the recent solar minimum, GCRs create persistent electric fields up to $\sim 700 \mathrm{~V} / \mathrm{m}$. We also find that large SEP events create transient but strong electric fields $\left(\geq 10^{6} \mathrm{~V} / \mathrm{m}\right.$ ) that may induce dielectric breakdown. Such breakdown would likely result in significant modifications to the physical and chemical properties of the lunar regolith within PSRs.

A variety of processes electrically charge the Moon's surface, with the dominant current sources typically being the photoemission of electrons by solar UV and the collection of charged particles from the surrounding plasma environment [Stubbs et al., 2014]. When the Moon is in the solar wind, these current sources typically charge the surface to a few volts positive on the dayside and down to $\approx 20-100 \mathrm{~V}$ negative near the terminator [Freeman and Ibrahim, 1975; Halekas et al., 2008]. On the upstream-facing side of the Moon, the lunar surface absorbs or reflects the incident solar wind plasma, thus forming a global-scale void or plasma wake downstream of the Moon [Ogilvie et al., 1996; Bosqued et al., 1996; Halekas et al., 2005]. The lunar surface within the wake region is mostly in shadow due to the solar wind's near-radial flow away from the Sun, so the surface charges to potentials of $\sim 200 \mathrm{~V}$ negative [Halekas et al., 2008]. Similar plasma wake structures likely occur locally on much smaller spatial scales downstream of topographic features, such as mountains and craters, near the terminator [Farrell et al., 2010]. Some studies predict that these so-called miniwakes charge areas within some permanently shadowed regions (PSRs) near the lunar poles up to $\sim 100 \mathrm{~V}$ negative [Zimmerman et al., 2011, 2012]. The Moon also passes through the Earth's magnetotail for a few days each month [e.g., see Stubbs et al., 2007], during which encounters with the plasma sheet region can charge the shadowed lunar surface to potentials of $\sim 200 \mathrm{~V}$ to $\sim 1 \mathrm{kV}$ negative [Halekas et al., 2008]. During large solar energetic particle (SEP) events the nightside lunar surface potential has been observed to reach a few kilovolts negative [Halekas et al., 2007, 2009].

While previous studies have only considered how these processes charge the uppermost layer of the lunar surface, we instead focus on deep dielectric charging of the regolith, in which energetic charged particles are deposited in the lunar subsurface. Because the lunar regolith is an electrical insulator (i.e., it has an extremely low conductivity), deposited charges can remain separated within it for prolonged intervals. Although deep dielectric charging has been an important topic for spacecraft engineering throughout the Space Age, the study presented here is the first to consider it as a lunar surface process. This process may have important consequences for the evolution of the regolith, particularly in PSRs where its electrical conductivity is especially low due to the extreme cold ( $\lesssim 100 \mathrm{~K}$ ). We have developed a one-dimensional, time-dependent model to estimate the deep dielectric charging driven by energetic charged particles at the Moon. Our model can apply to PSRs and other extremely cold regions on airless bodies throughout the solar system. 


\section{Properties of Energetic Charged Particles}

Galactic cosmic rays (GCRs) are relativistic ions and electrons that typically received their energy in supernova shocks [Krymskii, 1977; Axford et al., 1977; Bell, 1978; Blandford and Ostriker, 1978]. The GCR proton spectrum peaks at about $200 \mathrm{MeV}$, although some GCRs have energies many orders of magnitude greater. Near solar minimum, their flux is $\sim 4$ particles $\mathrm{cm}^{-2} \mathrm{~s}^{-1}$, and at solar maximum their flux is $\sim 2$ particles $\mathrm{cm}^{-2} \mathrm{~s}^{-1}$. This variation results from changes in the heliospheric magnetic field decreasing and increasing its shielding of the inner heliosphere [Smart and Shea, 1985]. About $97 \%$ of GCRs are protons and heavier ions, and the remainder are electrons [Smart and Shea, 1985]. Analysis of iron meteorites indicates that the variations in GCR fluxes over the past $1 \mathrm{Gyr}$ have been no more than a factor of 2 [Arnold et al., 1961]. Therefore, we can assume that past charging due to GCRs has likely varied little on long timescales. GCRs penetrate the regolith to depths on the order of tens of centimeters [Walker, 1980], and because they have a net positive charge, they necessarily charge the soil.

For GCR data, we use the Cosmic Ray Telescope for the Effects of Radiation (CRaTER) on board the Lunar Reconnaissance Orbiter (LRO) [Spence et al., 2010]. CRaTER began collecting data at the Moon on 23 June 2009. Because of its shielding, it detects only protons with energies greater than $\sim 15 \mathrm{MeV}$, but this energy regime includes the peak of the GCR spectrum.

Unlike GCRs, SEPs gain their energy in solar flares and the shocks of coronal mass ejections [Gosling, 1993; Reames, 2002]. Because electrons are much less massive, they propagate more quickly than the protons from the acceleration region [Van Hollebeke et al., 1975]. SEP events occur sporadically but more frequently during solar maximum, although major events can occur during solar minimum [Smart and Shea, 1985]. SEPs have lower characteristic energies than GCRs. While the ions have energies of $\sim 50 \mathrm{keV} /$ nucleon to $\sim 10$ $\mathrm{GeV} /$ nucleon and electrons have energies of $\sim 1 \mathrm{keV}$ to $\sim 10 \mathrm{MeV}$, both the ion and electron fluxes peak at energies lower than given in these ranges [McGuire and von Rosenvinge, 1984]. At the same time, however, SEP fluxes often exceed GCR fluxes by many orders of magnitude. Also, each event tends to have a unique energy spectrum, which limits our ability to generalize a typical SEP event.

For SEP proton and electron data, we use measurements from the Electron, Proton, and Alpha Monitor (EPAM) on the Advanced Composition Explorer (ACE) [Gold et al., 1998]. EPAM has been collecting data almost continuously since 30 August 1997. For the electrons, we use all four energy channels: 0.038-0.053, $0.053-0.103,0.103-0.175$, and $0.175-0.315 \mathrm{MeV}$. For the protons, we use all eight energy channels: $0.047-0.066,0.066-0.114,0.114-0.190,0.190-0.310,0.310-0.580,0.580-1.05,1.05-1.89$, and 1.89-4.75 MeV. Although the available SEP data do not cover all energies, EPAM covers a significant fraction of the typical SEP spectrum. SEPs at higher energies have much lower differential fluxes and so can be reasonably neglected. SEPs at lower energies have a larger differential flux but a smaller penetration depth. (For a further discussion of the implication of using EPAM data, see section 5.2.) Thus, using these data is sufficient for this initial study.

Deep dielectric charging by SEPs in the lunar subsurface depends on the relative penetration depths of the protons and electrons. If they both penetrate to roughly the same depths, then the net current density to the subsurface is determined by the differences of their respective current densities. Alternatively, if protons and electrons penetrate to different depths, then it is more appropriate to consider two separate subsurface charge layers, one accumulating a positive charge and the other a negative charge from the incident SEPs.

We estimate penetration depths of SEP protons and electrons using the National Institute of Standards' Stopping-Power and Range Tables for Protons (PSTAR) and for electrons (ESTAR). These tables estimate the ranges of protons and electrons by calculating the effects of electronic and nuclear collisions [International Commission on Radiation Units, 1993; Berger et al., 2005]. The ranges of these particles are available for various materials. Given how electrons scatter within matter and the issues associated with the EPAM energy ranges mentioned below, the overlap between the lower electron layer and upper proton layer will likely be greater than indicated in Figure 1 (see discussion in section 4).

Particle penetration distance predictions do not exist for either lunar regolith or lunar simulants. Therefore, we instead use silicon dioxide $\left(\mathrm{SiO}_{2}\right)$ as a regolith proxy, because lunar regolith comprises mainly silicate material, i.e., mainly silicon and oxygen [Papike et al., 1991]. (Note that the chemical composition of the soil has little effect on the penetration of energetic charged particles. For example, the penetration distance would increase by less than $10 \%$ if the soil were pure aluminum.) The PSTAR and ESTAR ranges are given 


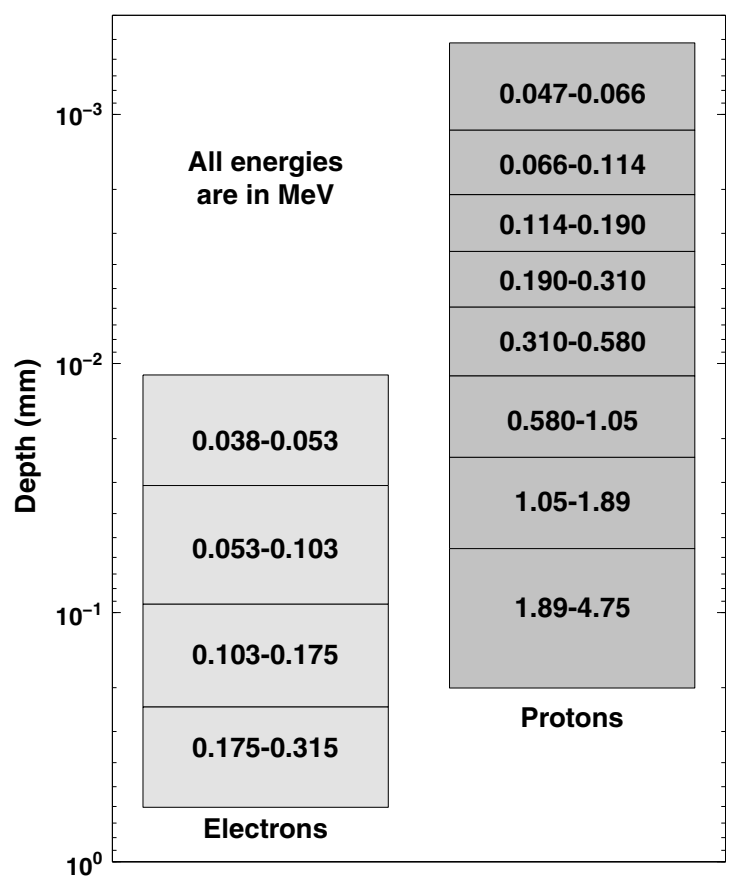

Figure 1. The typical penetration depths of the (left) electron and (right) proton energy channels detected by ACE/EPAM. The more energetic particles penetrate deeper. in terms of the mass column density $\sigma_{m}$ $\left(\mathrm{g} / \mathrm{cm}^{2}\right)$. We convert these to penetration distances by assuming a lunar soil density of $\rho_{m}=1.5 \mathrm{~g} / \mathrm{cm}^{3}$, a value agreeing with the best estimates for the mean density of the soil's upper 0-15 cm [Carrier et al., 1991]. For a given stopping range, the penetration distance within the soil is $\sigma_{m} / \rho_{m}$, which is also the penetration depth for a normally incident particle. This depth, however, is not the typical penetration depth, because both GCRs and SEPs arrive from $2 \pi$ sr. The particle's angle of incidence determines the depth to which it penetrates:

$$
z=\frac{\sigma_{m}}{\rho_{m}} \cos \theta
$$

where $\theta$ is the angle of incidence with respect to the normal.

As mentioned, we assume that the source of energetic particles subtends $2 \pi \mathrm{sr}$, a good assumption within larger complex craters and impact basins (diameters of tens to hundreds of kilometers), where the crater rim is typically low on the horizon, if visible at all [Pike, 1974]. The rims of smaller (diameters $<15 \mathrm{~km}$ ) simple craters, however, block from view about a third of the sky. These rims reduce proportionally the incident particle fluxes of energetic particles, thus modifying the depth distribution. Therefore, our model results will be most relevant to larger craters.

With an isotropic distribution of incident particles, the median penetration depth $z_{m}$ has an equivalent incidence angle of $\theta=60^{\circ}\left(\cos 60^{\circ}=0.5\right.$ ). That is, half the incoming particles have $\theta<60^{\circ}$ and are deposited below $z_{m}$, while the other half have $\theta>60^{\circ}$ and are deposited above $z_{m}$. Therefore, we take the typical penetration depth of a particle with a given energy to be $z_{m}$. The results are shown on a log scale in Figure 1. Although some of the observed protons can penetrate to $0.2 \mathrm{~mm}$, seven of the eight proton channels of ACE/EPAM stop within the first $0.1 \mathrm{~mm}$. Protons from only three of the eight channels penetrate as deeply as the electrons. The electrons, on the other hand, can penetrate to $0.6 \mathrm{~mm}$. As there is a significant difference in the proton and electron penetration depths at SEP energies, we assume that they form two separate charge layers in the lunar subsurface. As mentioned above, the scattering of the electrons means that we are likely overestimating their penetration depth. Our model, however, examines both limits_full overlap and no overlap-so the exact penetration depth is unimportant, as shown in section 4.

Note that we consider only the charging from charged particles added to, not removed from, the regolith. Atomic nuclei, for instance, can be ejected, or spalled, by GCRs. Atomic nuclei are less likely, however, to escape than are secondary electrons, because GCRs are much less likely to have nuclear interactions than electronic ones [Vaniman et al., 1991]. These secondary electrons escape more easily if created near the regolith's surface, which is most likely for GCRs (and SEP ions) with grazing incidences. Therefore, the escape of these electrons from near the surface likely charges the upper, positive layer even more positive, reinforcing the charging we find with the model. Note, too, that the yield of secondary electrons due to incident SEP electrons is probably negligible, as shown by studies using Apollo samples of the lunar regolith [Willis et al., 1973]. All these phenomena are either insignificant or reinforce the charging we estimate below with the model.

\section{Electrical Properties of the Lunar Regolith}

As we have just described, when energetic particles penetrate the regolith, they deposit space charge within it. As described in the previous section, we assume that the particles deposit their charge at their penetration depth. The regolith acts as a capacitor, such that with time, the space charge dissipates, thus reducing 
the overall electric field. During this dissipation, the regolith also functions as a resistor. Therefore, we can treat the regolith as a "leaky" capacitor, in which the charge density $\rho$ decays from its initial value $\rho_{0}$ as described by

$$
\rho(t)=\rho_{0} e^{-t / \tau},
$$

where $t$ is time and $\tau$ is the $e$-folding time for discharging [see, for example, Buhler et al., 2007]. This $e$-folding time is the ratio of its permittivity $\epsilon$ to its conductivity $\sigma: \tau=\epsilon / \sigma$. (Permittivity is the effectiveness of a material at keeping charge carriers within it separated, and conductivity is its effectiveness at allowing current to flow through it.) Finding the value for $\tau$ is necessary for estimating deep dielectric charging of the lunar regolith.

Both the permittivity and conductivity of the lunar sample soils have been measured in laboratory experiments. Olhoeft and Strangway [1975] reviewed 92 laboratory measurements of the dielectric permittivity and found the soil's dielectric constant (or relative permittivity) to be between $\sim 1.5$ and 4 for the densities characteristic of the upper regolith, with the most common value being $\approx 2$. We therefore assume $\epsilon=2 \epsilon_{0}$ for this study.

Olhoeft et al. [1974] showed that an Apollo 15 soil sample's conductivity has a temperature dependence given by

$$
\sigma=6 \times 10^{-18} e^{0.0237 T},
$$

where $\sigma$ is in units of siemens per meter $(\mathrm{S} / \mathrm{m})$ and $T$ is the temperature in kelvin. While any given location on the Moon may have a different conductivity due to mineralogical and physical variations of the near-surface regolith, we assume the regolith in PSRs to follow the above temperature relation.

The above form for the conductivity applies only if the charging occurs over a vertical extent that is much greater than the typical grain size. In other words, the dissipation current must pass through many grains for that equation to apply. Otherwise, we would have to treat the soil as comprising individual rocks, which have about 4 orders of magnitude higher conductivity [Olhoeft et al., 1973]. According to the review by McKay et al. [1991], the average grain size for lunar soil is between 60 and $80 \mu \mathrm{m}$, although this is likely an overestimate due to the size-sorting sieving technique being limited to grain sizes $>10 \mu \mathrm{m}$. Aerosol measurements have shown that grain sizes can be as small as a few $100 \mathrm{~nm}$ [Greenberg et al., 2007]. Thus, based on the penetration depths we have shown, protons detected by EPAM stop within the first few grains, while all electrons stop by tens of grains deeper in the regolith. Therefore, the particle penetration and charge separation occur over a depth much greater than the typical grain size, and the form for the conductivity given by equation (3) is valid. Note, too, that we define the penetration depth to be the depth at which half the particles have already stopped (i.e., the center of the charge layer), so the other half penetrates deeper.

As stated above, the electrical conductivity of the regolith in PSRs depends on the temperature. Observations using the Diviner instrument on LRO show typical temperatures to be $\sim 50 \mathrm{~K}$ [Paige et al., 2010]. According to equation (3), this implies that the soil within PSRs has a conductivity of about $10^{-17} \mathrm{~S} / \mathrm{m}$.

For the above values of the regolith's permittivity and conductivity, the discharging timescale $\tau$ within PSRs is $\sim 20$ days, which is on the order of a lunation. Because $\epsilon$ can be as great as 4 [Olhoeft and Strangway, 1975], $\tau$ may even be as long as 40 days. As we will show in section 5 , this timescale determines the strength of the electric fields that form in response to the accumulation of energetic charged particles.

\section{Double Charge Layer Model}

To estimate the electric fields due to deep dielectric charging by energetic particles at the Moon, we have developed a one-dimensional, time-dependent model. For this first-order model, we ignore all input currents other than the energetic charged particles. As we limit the model to PSRs, we can ignore currents from the photoemission of electrons. Similarly, we consider areas within PSRs where the wake-modified ambient plasma current can reasonably be neglected [Farrell et al., 2010]. Therefore, we adopt the common assumption that the environment within PSRs can effectively be treated as an ideal vacuum. (Note also that the environment above lunar nightside areas shielded by magnetic anomalies could also be treated as an ideal vacuum, and so these areas could be favorable locations for deep dielectric charging [cf. Bamford and et al., 2012].) We also assume the regolith to be an ohmic material; in other words, the regolith's charging decay 
is exponential, as in equation (2) (note that granular materials may deviate from this assumption, as experiments with lunar simulant seem to indicate [Buhler et al., 2007]). We ignore radiation-induced conductivity, caused by significant radiation damage increasing the medium's conductivity. Properly accounting for this effect requires laboratory measurements [Frederickson, 1977], but we know of no such studies for lunar soil. Also, because the regolith's nonlinear polarization terms, which would come into effect at very strong electric fields, are unknown, we do not include them. We further assume that the gain or loss in energy of the energetic particles as they pass through the potential difference between the surrounding space environment and the lunar surface is negligible (see sections 1 and 2). We also assume the charge deposition layer to be infinitely planar [cf. Stubbs et al., 2014]. This is reasonable because the particle deposition occurs over regions whose length scales $L$ are very large relative to the penetration depths (i.e., $z \ll L$ ). According to this approximation, the electric field due to a given charged layer is symmetric and uniform. Therefore, unless both charge species penetrate to identical depths (as discussed in section 2), the exact penetrations depths do not affect the resulting electric fields. This is analogous to a parallel plate capacitor.

The model works as follows. Two current densities penetrate the regolith: one of energetic protons $\left(J_{\mathrm{EP}}^{+}\right)$and another of electrons $\left(J_{\mathrm{EP}}^{-}\right)$. (The subscript EP refers to energetic particles.) The input currents create two layers of space charge within the regolith: an upper, positive layer and a lower, negative layer. For convenience, we assume that the layers do not overlap, as discussed in section 2. From Gauss's law, the electric fields due to these two infinite charged slabs are

$$
\begin{aligned}
& E^{+}=\frac{\kappa^{+}}{2 \epsilon} \\
& E^{-}=\frac{\kappa^{-}}{2 \epsilon}
\end{aligned}
$$

where $\kappa$ is the areal charge density of the layers, in units of $\mathrm{C} \mathrm{m}^{-2}$ (for brevity, we shall subsequently refer to the areal charge density of the layers as simply the "charge density"). The superposition of these two electric fields creates three regions: (i) within the regolith at the surface, i.e., at the top of the upper charge layer; (ii) in the gap between the charge layers; and (iii) in the interior below the lower charge layer. These electric fields are given by

$$
\begin{gathered}
E_{\text {surface }}=E^{+}+E^{-}=\frac{\kappa^{+}+\kappa^{-}}{2 \epsilon} \\
E_{\text {gap }}=-E^{+}+E^{-}=\frac{-\kappa^{+}+\kappa^{-}}{2 \epsilon} \\
E_{\text {interior }}=-E^{+}-E^{-}=\frac{-\left(\kappa^{+}+\kappa^{-}\right)}{2 \epsilon},
\end{gathered}
$$

where the positive direction is away from the Moon's interior. (A consequence of this model is that $\kappa^{+} \geq 0$ and $\kappa^{-} \leq 0$.)

We assume that no charge flows out of the surface, meaning charge only dissipates within the gap region and toward the interior below the lower layer. The gap current density $J_{\text {gap }}$ dissipates charge buildup by transporting charge between the two layers. The interior current density $J_{\text {interior }}$ dissipates charge through a much larger scale electrical circuit in the regolith that is not explicitly considered in this study. Electrons, whether provided by that circuit or by $J_{E P^{\prime}}^{-}$are assumed to be the sole charge carriers for $J_{\text {gap }}$ and $J_{\text {interior }}$. These two dissipation current densities are

$$
\begin{gathered}
J_{\text {gap }}=\sigma E_{\text {gap }} \\
J_{\text {interior }}=\sigma E_{\text {interior }}
\end{gathered}
$$

All of these quantities are shown in Figure 2 for the four cases considered here.

The rate of change of the two layers' charge densities is

$$
\begin{gathered}
\frac{\mathrm{d} \kappa^{+}}{\mathrm{d} t}=J_{\mathrm{EP}}^{+}(t)+J_{\text {gap }}(t) \\
\frac{\mathrm{d} \kappa^{-}}{\mathrm{d} t}=J_{\mathrm{EP}}^{-}(t)-J_{\text {gap }}(t)+J_{\text {interior }}(t)
\end{gathered}
$$



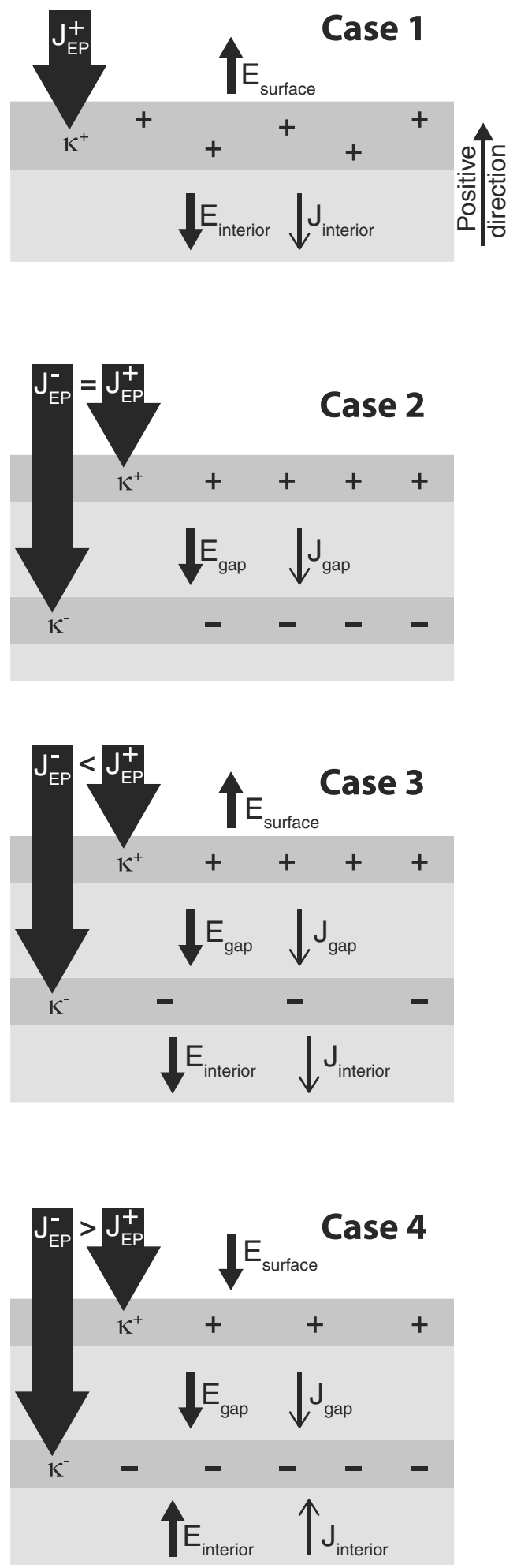

Figure 2. This cartoon shows the electric fields and dissipation current densities for the four possible subsurface charging cases: (1) only GCRs $\left(J_{\mathrm{EP}}^{+}>0\right.$; $\left.J_{\mathrm{EP}}^{-}=0\right)$, (2) SEP protons and electrons with equal number fluxes $\left(\left|J_{E P}^{+}\right|=\left|J_{E P}^{-}\right|\right)$, (3) SEP protons with a greater number flux than the electrons $\left(\left|J_{E P}^{+}\right|>\left|J_{E P}^{-}\right|\right)$, and (4) SEP protons with a smaller number flux than the protons $\left(\left|J_{E P}^{+}\right|<\left|J_{E P}^{-}\right|\right)$. where $J_{E P}^{+}(t)$ and $J_{E P}^{-}(t)$ can be input into the model as time series derived from spacecraft measurements. In other words, the upper, positively charged layer is dissipated by only the gap current density, while the lower, negative layer is affected by both the gap and interior current densities.

In this study, the initial conditions for the model at $t=t_{0}$ are set to $\kappa^{+}\left(t_{0}\right)=0$ and $\kappa^{-}\left(t_{0}\right)=0$, which means that all the electric fields start at zero, as dictated by equations (6)-(8). At $t=t_{0}$, the energetic particle currents, $J_{\mathrm{EP}}^{+}\left(t_{0}\right)$ and $J_{\mathrm{EP}}^{-}\left(t_{0}\right)$, begin flowing, and charge starts accumulating in the upper and lower layers according to equations (11) and (12). At the next time step $\left(t=t_{1}\right), \kappa^{+}\left(t_{1}\right)$ and $\kappa^{-}\left(t_{1}\right)$ are nonzero. The resulting electric fields are calculated using equations (6)-(8), and these fields determine the dissipation currents using equations (9) and (10). The resulting values of $J_{\text {gap }}\left(t_{1}\right)$ and $J_{\text {surface }}\left(t_{1}\right)$ are then fed into the next iteration, along with $J_{\mathrm{EP}}^{+}\left(t_{1}\right)$ and $J_{\mathrm{EP}}^{-}\left(t_{1}\right)$, to determine the change in $\kappa^{+}$and $\kappa^{-}$using equations (11) and (12). Each iteration continues likewise. By following the same approach, the model can be started with any set of initial conditions.

In every simulation, the time step $\Delta t$ is much shorter than the discharging timescale $(\Delta t \ll \tau)$. If this condition was satisfied, we found the simulations to be insensitive to the choice of $\Delta t$ by comparing them with analytical solutions for the most basic cases. In the data-driven cases, $\Delta t$ is simply the resolution of the input time series $J_{\mathrm{EP}}^{+}$and $J_{\mathrm{EP}}^{-}$.

Assuming that EPAM detects all SEPs, $E_{\text {gap }}$ is the strongest possible electric field, given that the input current densities $J_{E P}^{+}$and $J_{E P}^{-}$are the only means of charging the regolith. This is because it results from assuming that all protons and electrons are deposited in two nonoverlapping layers. If the upper and lower layers partially overlap each other, then $E_{\text {gap }}$ would be reduced because the overlap would cancel some of the charging in that region. (The exact reduction would depend on the depth profile of both layers.) If the layers completely overlap each other, then there is no gap, so $E_{\text {gap }}=0$. As can be seen in Figure 1, the layers partially overlap, so the maximum electric field strength within the charging layers is less than $\left|E_{\text {gap }}\right|$. Conversely, $E_{\text {surface }}$ and $E_{\text {interior, }}$ which are equal and opposite $\left(E_{\text {surface }}=-E_{\text {interior }}\right)$, set the lower limit on the electric field magnitude in the lunar 


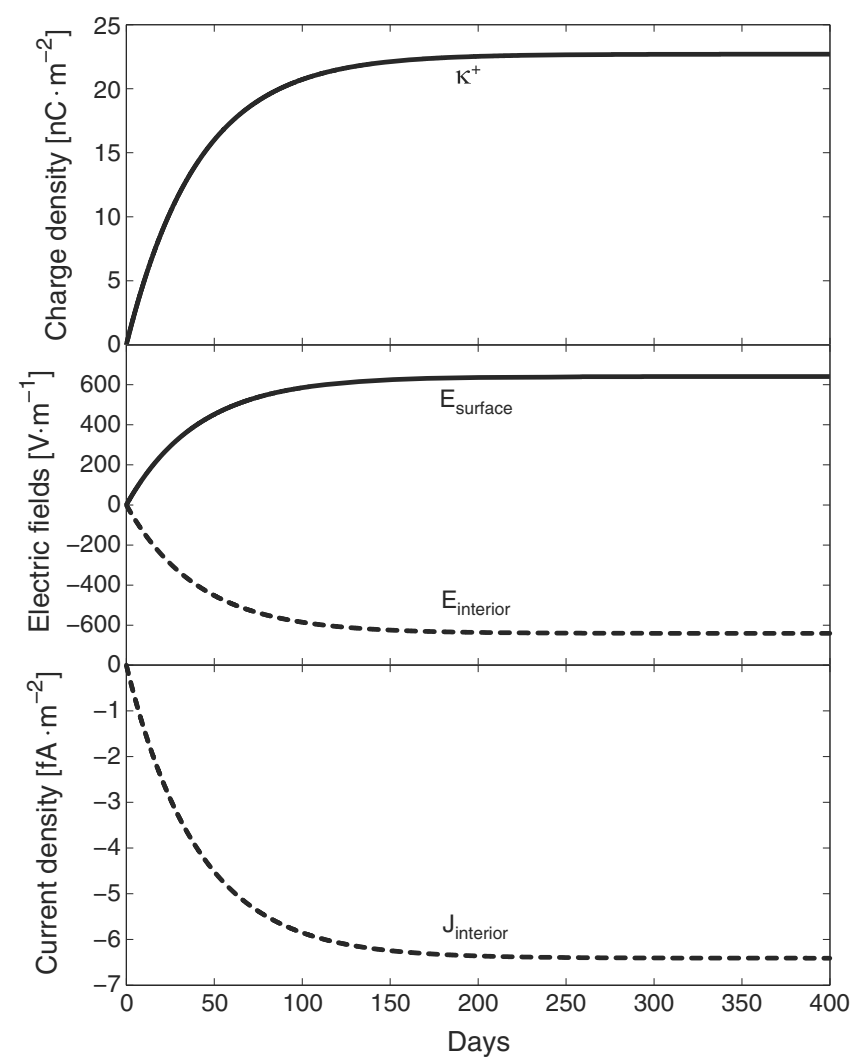

Figure 3. Model output using constant flux of protons only (based on average GCR proton fluxes during solar minimum). (top) The charge density due to the accumulation of protons. (middle) The surface (solid line) and interior (dashed) electric fields resulting from the charged layer. (bottom) The interior current density dissipating the charged layer.

regolith. They are independent of whether a gap exists between the upper and lower charge layers. Instead, as equations (6) and (8) show, these electric fields depend only on the net charge in both layers $\left(\kappa^{+}+\kappa^{-}\right)$. This is because, in the regions above and below them, the two charge layers appear to exist just as a single layer. Note that adding a surface charge layer is unlikely to prevent this subsurface charging, since a third layer cannot simultaneously decrease or nullify all three electric fields.

Therefore, for a given set of $J_{E P}^{+}$and $J_{E P}^{-}$, the electric field within the regolith can be no stronger than $\left|E_{\text {gap }}\right|$ and no weaker than $\left|E_{\text {surface }}\right|$ (or $\left|E_{\text {interior }}\right|$ ). This is only true, however, if EPAM detects SEPs at all relevant energies. In the next section, we discuss how this assumption affects our calculations.

\section{Model Results}

\subsection{Test Case Examples}

To demonstrate the model, we run three test cases. (In all modeling simulations, whether a test case or not, we start with no subsurface charge, i.e., $\kappa^{+}=\kappa^{-}=0$, as discussed in section 4.) The first case is for a constant proton flux of 4 particles $\mathrm{cm}^{-2} \mathrm{~s}^{-1}$, such as that typically observed for GCRs during solar minimum (see section 2). Case (1) in Figure 2 illustrates this scenario. There is no second, deeper charged layer; all the charge is deposited in a single layer $\left(J_{\mathrm{EP}}^{-}\right.$and $\kappa^{-}$are both set to zero). We run the model for 400 days ( 20 discharging timescales). The model results are shown in Figure 3 for a dielectric constant of two and a typical PSR regolith conductivity of $10^{-17} \mathrm{~S} / \mathrm{m}$. The charge density (top row) increases with time, until the resulting electric field (middle row) is strong enough to create a dissipation current (bottom row) able to remove the space charge at the rate it is added (from equations (11) and $12, J_{\mathrm{EP}}^{+}+J_{\text {interior }}=0$ ). That equilibrium electric field is $640 \mathrm{~V} / \mathrm{m}$.

A second, though unrealistic, scenario occurs if the fluxes of protons and electrons are equal (4 particles $\mathrm{cm}^{-2} \mathrm{~s}^{-1}$ ), with the electrons penetrating more deeply, as is the case for the SEPs detected by ACE/EPAM (see the second case in Figure 2). The model results are shown in Figure 4. In this situation, the amount of charge 


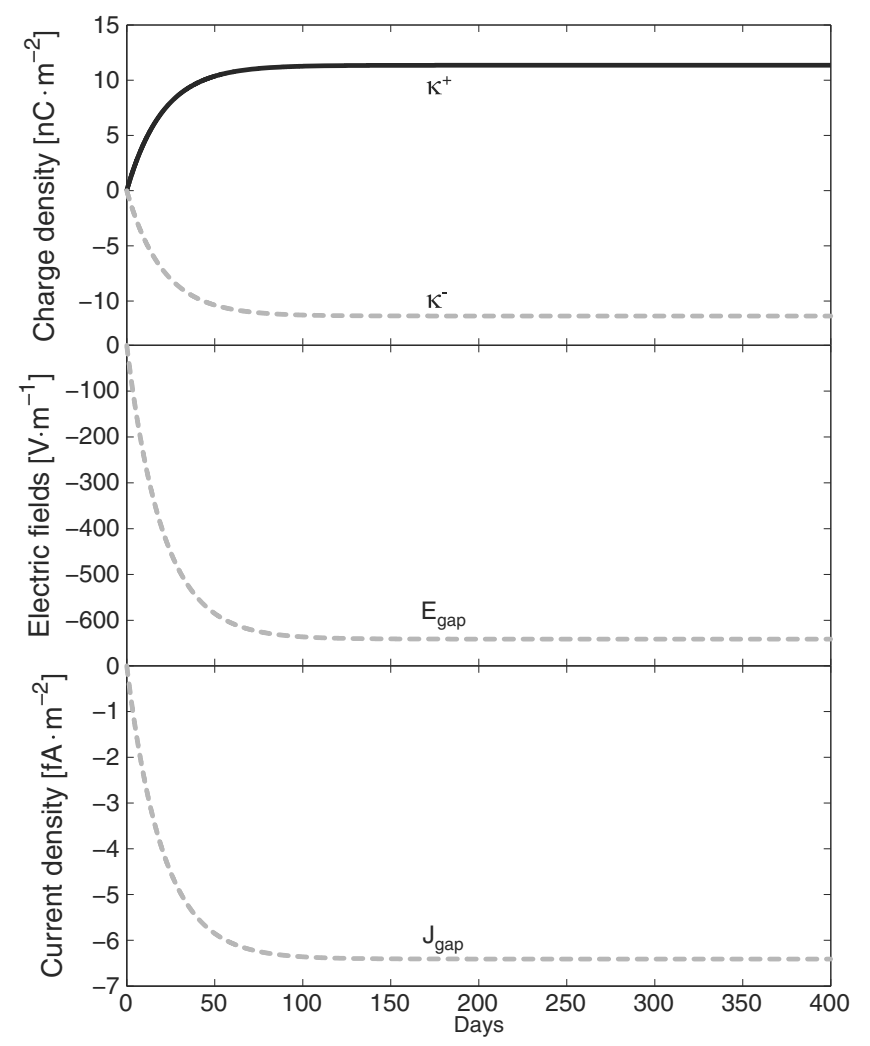

Figure 4. Model output using constant and equal fluxes of protons and electrons. (top) The charge density due to the accumulation of protons (solid line) and electrons (gray dashed line). (middle) The gap electric field resulting from the charged layers. (bottom) The gap current density dissipating the two layers. (Throughout all subsequent figures, gap electric fields and gap current densities are shown as gray dashed lines.)

accumulated in the upper (positive) and lower (negative) charge layers increases at the same rate. Therefore, the surface and interior electric fields remain zero at all times, because the net charge of the region including both layers is zero. Between the layers, however, the gap electric field also increases with increasing charging. Eventually, $E_{\text {gap }}$ is sufficient to drive a $J_{\text {gap }}$ that dissipates the charges at their accumulation rate. For the assumed proton and electron fluxes, $E_{\text {gap }}=640 \mathrm{~V} / \mathrm{m}$, which is the same as in the first scenario. Initially, both the gap field and current density increase more quickly than in the first scenario. This increase, however, causes the layers to dissipate charge more quickly, until reaching an equilibrium in which the charge density in the positively charged upper layer is half that in the first scenario. This results in the same equilibrium electric field strength. While the electric field was outside the single positively charged layer in the first scenario, in this second scenario the electric field occurs only within the gap.

The final test case is the same as the second but with a higher electron flux (40 particles $\mathrm{cm}^{-2} \mathrm{~s}^{-1}$ ). The proton flux is still 4 particles $\mathrm{cm}^{-2} \mathrm{~s}^{-1}$. This case is illustrated by the fourth case of Figure 2 and simulates a relatively weak SEP event in which more electrons than protons impact the Moon. Figure 5 shows the results.

This scenario is more complex than the previous two. Initially, the upper layer charges positively and the lower negatively, as expected. The magnitude of the negative layer's charge density, however, increases more rapidly. The gap electric field is directed downward, thus causing the upper layer to dissipate positive charge faster than it accumulates (i.e., $\left|J_{\text {gap }}\right|>\left|J_{E P}^{+}\right|$), such that it begins to charge negatively. Eventually, the system reaches equilibrium. A dissipation current still exists within the gap; it is carried by the electrons in the regolith material and cancels out the charge from the energetic protons accumulated in the upper layer. As we would expect, the electric field driving this current has a magnitude of $640 \mathrm{~V} / \mathrm{m}$, just as in the first test case. The electric fields at the surface and in the interior, however, are nearly $6 \mathrm{kV} / \mathrm{m}$.

\subsection{Data-Driven GCR and SEP Simulations}

The above test case scenarios demonstrate how this relatively basic lunar deep dielectric charging model responds to a variety of steady boundary conditions. We now use data from CRaTER and ACE/EPAM to 


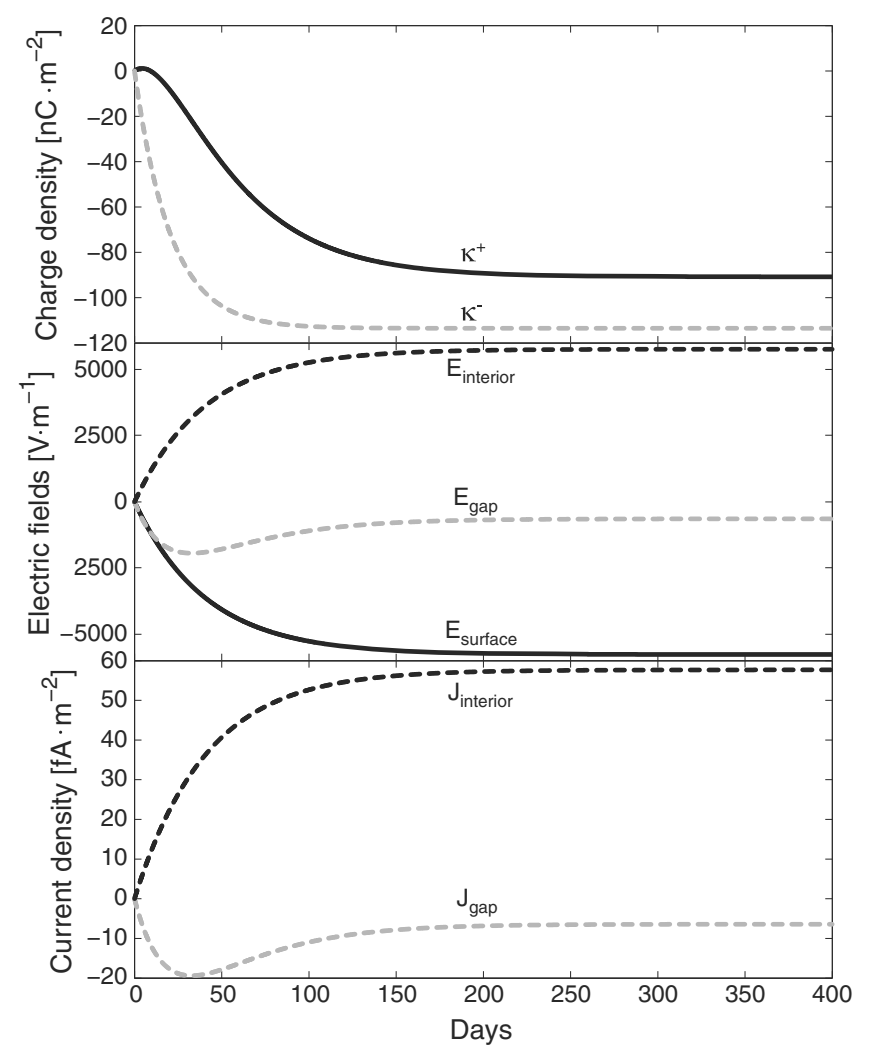

Figure 5. Model output using constant fluxes of protons and, at an order of magnitude greater flux, electrons. (top) The charge density due to the accumulation of protons (solid line) and electrons (gray dashed line). (middle) The surface (solid line), gap (gray dashed), and interior (dashed) electric fields resulting from the charged layers. (bottom) The gap (gray dashed line) and interior (dashed) current densities dissipating the two layers.

estimate the subsurface electric fields within lunar PSRs. We begin with GCR data and then run the model for three large SEP events.

Our first step in calculating PSR subsurface electric fields is to estimate those due to GCRs detected by CRaTER (this corresponds to the first hypothetical scenario in section $5 \mathrm{a}$, in which $J_{\mathrm{EP}}^{-}$and $\kappa^{-}$are both zero). As before, we initialize the simulation with zero subsurface charge, so we expect the first several months of the simulation to be unrealistic as it approaches toward a near-equilibrium state. We use particles triggering both D1 and D2, which are neighboring detectors in the telescope stack; this detection criterion corresponds to a geometric factor of $24.152 \mathrm{~cm}^{2}$ sr [Spence et al., 2010]. The resulting GCR flux is shown in Figure 6 (first row). We show data only up until March 2011, because SEP events contaminate most of the GCR data after that period. The GCR flux reached an average value of nearly 4.5 particles $\mathrm{cm}^{-2} \mathrm{~s}^{-1}$ during the most recent solar minimum near the beginning of 2010. From this GCR flux, we derive the current density, $J_{E P}^{+}$, which is the CRaTER number flux multiplied by the proton's charge. After 2010, the Sun's activity has increased, causing a decrease in GCR flux. This has caused the resulting charge density, electric field strengths, and current density magnitudes to decrease as well (see second to fourth rows in Figure 6). The peak electric fields $(700 \mathrm{~V} / \mathrm{m})$ occurred near the end of solar minimum in this simulation. As the GCR flux decreased, the electric field magnitude in the regolith also decreased to less than $600 \mathrm{~V} / \mathrm{m}$. This agrees with the results of the first test scenario above. (Note that these peak fields apply above and below the region of charging; due to the typical penetration of GCRs, this region is the top $\sim 40 \mathrm{~cm}$ of regolith. Within the charging region, the field will be weaker.)

The first SEP event we analyze is the Bastille Day storm in July 2000 (Figure 7). The ACE/EPAM proton and electron fluxes, shown in the first row, are used to calculate the model input current densities, $J_{E P}^{+}$and $J_{E P^{\prime}}^{-}$ respectively. The peak fluxes were $1.4 \times 10^{6}$ protons $\mathrm{cm}^{-2} \mathrm{~s}^{-1}$ and $3.9 \times 10^{6}$ electrons $\mathrm{cm}^{-2} \mathrm{~s}^{-1}$, and the particle fluences over the month of July were $5.6 \times 10^{13}$ protons $/ \mathrm{cm}^{2}$ and $1.8 \times 10^{14}$ electrons $/ \mathrm{cm}^{2}$. 


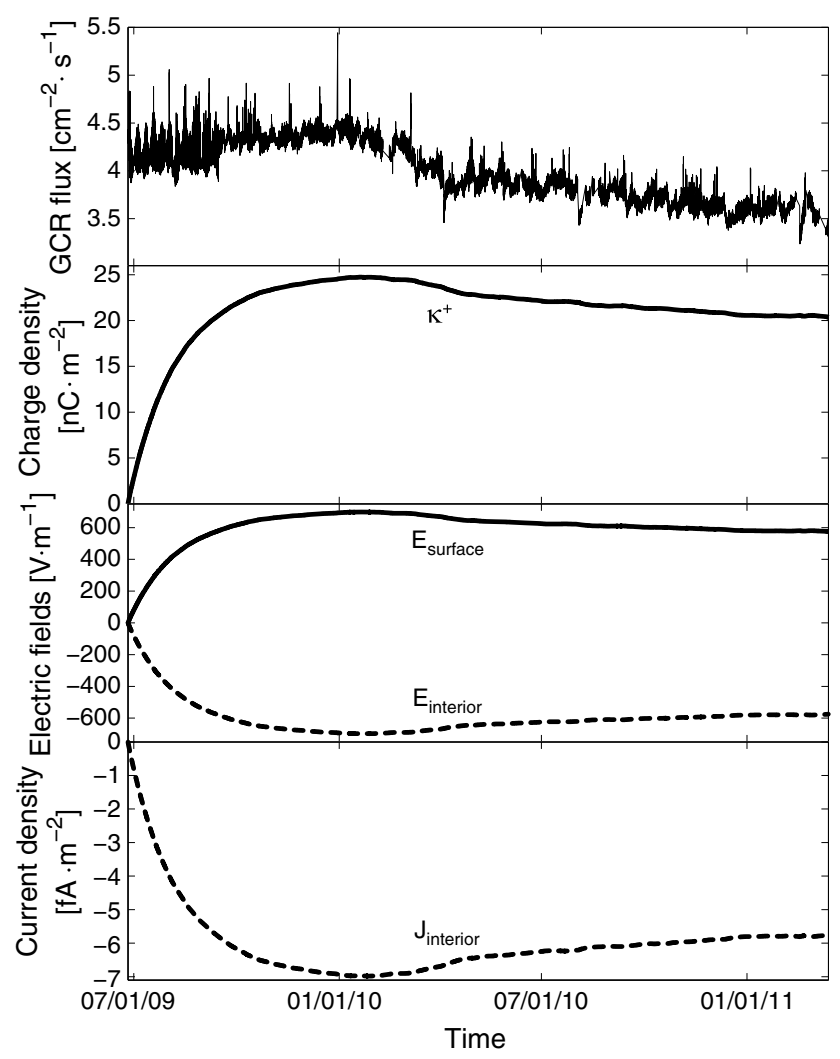

Figure 6. Model outputs using (first row) GCR fluxes measured by CRaTER. Note that the simulation starts with zero charge density and takes a few months to reach realistic (near-equilibrium) values; the variations due to GCR fluctuations occur after that time. (second row) The charge density due to the accumulation of protons. (third row) The surface (solid line) and interior (dashed) electric fields resulting from the charged layer. (fourth row) The interior current density dissipating the charged layer.

At the start of the event, on 13 July, the proton flux was greater than the electron flux, but the electron flux became dominant as the event progressed. The electric field magnitude peaked in the gap (13 MV/m), while the surface and interior fields had magnitudes of $7.2 \mathrm{MV} / \mathrm{m}$. After the event, the charge densities, fields, and current densities all began to decay.

As mentioned in the previous section, based on our assumption, these two field strengths could be considered the upper and lower limits of the electric field for the given set of $J_{E P}^{+}$and $J_{E P}^{-}$(this is also true for the following two events). $J_{\mathrm{EP}}^{+}$and $J_{\mathrm{EP}}^{-}$, however, do not include all SEPs because ACE/EPAM does not detect SEPs at all energies. Higher-energy SEPs are an insignificant fraction of the total flux, so we can reasonably ignore them. Lower energy SEPs, however, have a greater flux and shallower penetration depth. Including these lower energy SEPs in the model would increase $\left|E_{\text {gap }}\right|$, but only because that field strength assumes the charge layers do not overlap. In reality, however, the peak electric field strength within the charged layers could be greater than, less than, or equal to $\left|E_{\text {gap }}\right|$, depending on the particle fluxes at each depth within the regolith. On the other hand, $E_{\text {surface }}$ and $E_{\text {interior }}$ depend only on the difference between $J_{\mathrm{EP}}^{+}$and $J_{\mathrm{EP}}^{-}$, i.e., on the net charge deposited to the subsurface. The electrons dominate during this event (and the two below), despite being detected over an energy range $(0.038-0.315 \mathrm{MeV})$ that is an order of magnitude smaller than the proton's range $(0.047-4.75 \mathrm{MeV})$. It is therefore likely that the electrons would dominate even at lower energies (i.e., higher fluxes). This means that including lower energy SEPs would make $\left|E_{\text {surface }}\right|$ and $\left|E_{\text {interior }}\right|$ greater than estimated by this model.

The second SEP event we consider occurred in November 2001 (Figure 8). The peak fluxes were $1.9 \times 10^{6}$ protons $\mathrm{cm}^{-2} \mathrm{~s}^{-1}$ and $5.0 \times 10^{6}$ electrons $\mathrm{cm}^{-2} \mathrm{~s}^{-1}$, and the particle fluences over this time period were $1.9 \times 10^{14}$ protons $/ \mathrm{cm}^{2}$ and $3.8 \times 10^{14}$ electrons $/ \mathrm{cm}^{2}$. The strongest electric field occurred in the gap, with a value of $16 \mathrm{MV} / \mathrm{m}$. The interior and surface fields had peak magnitudes of $7.8 \mathrm{MV} / \mathrm{m}$. 


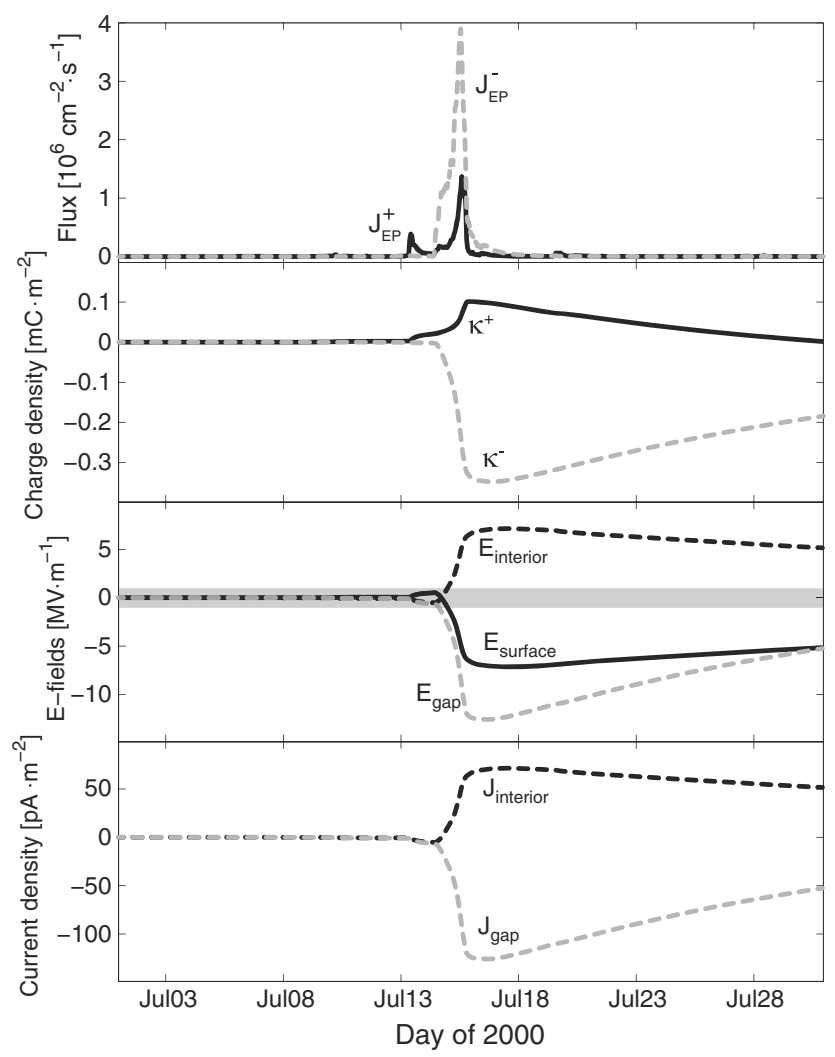

Figure 7. Model outputs using (first row) SEP proton (solid line) and electron (gray dashed line) data from ACE/EPAM during July 2000. (second row) The charge density due to the accumulation of protons (solid line) and electrons (gray dashed line). (third row) The surface (solid line), gap (gray dashed), and interior (dashed) electric fields resulting from the charged layers. The gray bar shows the range within which dielectric breakdown does not occur (see section 6). (fourth row) The gap (gray dashed line) and interior (dashed) current densities dissipating the two layers.

Finally, the Halloween storms of 2003 are shown in Figure 9. Unlike the two previous SEP events, the protons had a higher peak flux than the electrons: $2.1 \times 10^{6}$ protons $\cdot \mathrm{cm}^{-2} \cdot \mathrm{s}^{-1}$ and $1.1 \times 10^{6}$ electrons $\cdot \mathrm{cm}^{-2} \cdot \mathrm{s}^{-1}$. The electrons, however, had a greater fluence over this time period: $1.3 \times 10^{14}$ protons $\cdot \mathrm{cm}^{-2}$ and $1.8 \times 10^{14}$ electrons $\cdot \mathrm{cm}^{-2}$. The strongest electric field occurred in the gap, with a value of $8.8 \mathrm{MV} / \mathrm{m}$. The interior and surface fields had magnitudes of $2.6 \mathrm{MV} / \mathrm{m}$. The electric fields created by all three of these SEP events were strong enough that they may have induced dielectric breakdown.

\section{Dielectric Breakdown}

The subsurface electric field strengths our model predicts during large SEP events pass the threshold necessary for dielectric breakdown. Much research on how energetic particles charge dielectrics has occurred over the past 50 years within the spacecraft engineering community (see the reviews by Whipple [1981] and Robinson and Coakley [1992]). The deep dielectric charging of a spacecraft can affect the operation of instruments and, in extreme cases, results in dielectric breakdown (see the reviews by Frederickson [1983] and Balmain [1987]). For example, the Internal Discharge Monitor on board the Combined Release and Radiation Effects Satellite detected dielectric breakdown while in Earth's radiation belts at a fluence of $\sim 2 \times 10^{10}$ electrons $/ \mathrm{cm}^{2}$ [Violet and Frederickson, 1993; Frederickson et al., 1992]. Electrostatic discharges are the leading cause of space mission failures due to the particle radiation environment within the magnetosphere [Bedingfield et al., 1996; Koons et al., 1998].

Dielectric breakdown occurs when the electric field within an insulator is great enough to cause the material to ionize and form a gaseous conducting channel [Budenstein, 1980]. The shape of such a channel resembles a tree, with a main stem and small filaments branching off it. Depending on the strength of the field, the channel can penetrate completely through the dielectric (full breakdown) or only partially (partial breakdown) [Frederickson et al., 1986]. For the purposes of this paper, we use the term "breakdown" to refer to 


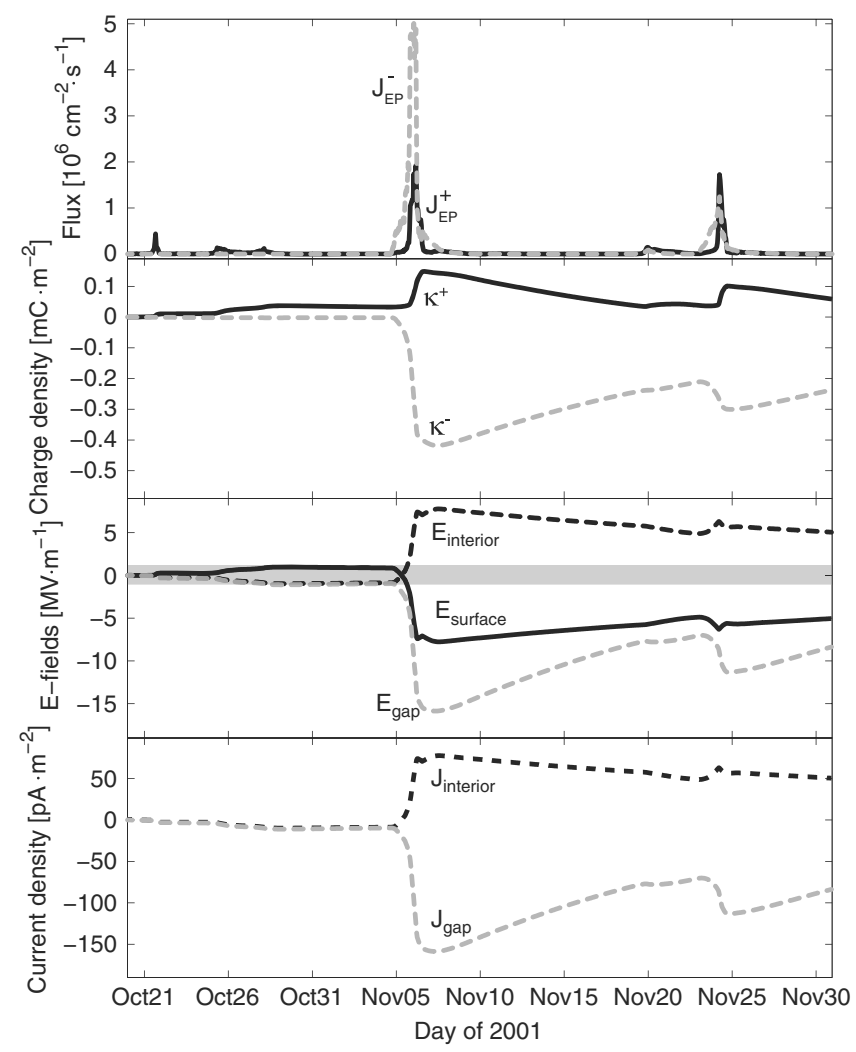

Figure 8. Same as Figure 7 but for SEP events during November 2001.

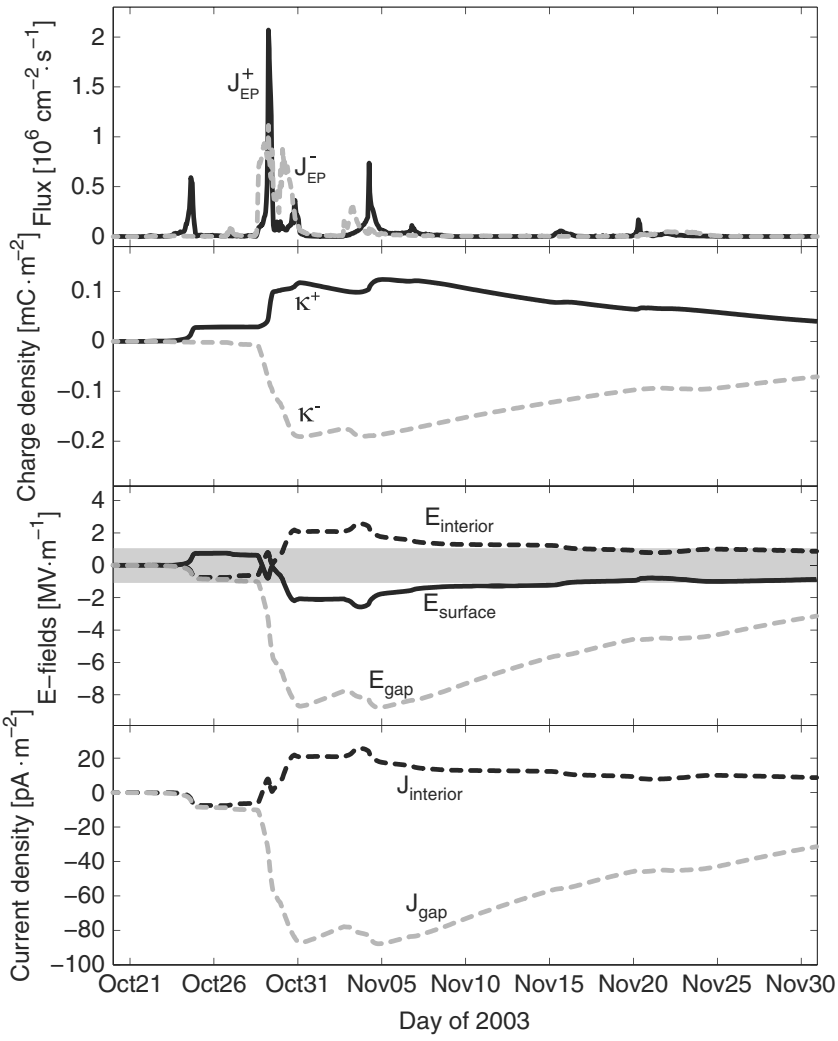

Figure 9. Same as Figure 7 but for SEP events during October and November 2003. 
both, since breakdown channels occur in both situations. To initiate full breakdown, the electric field must be $\sim 10^{7} \mathrm{~V} / \mathrm{m}$, while partial breakdown can occur at fields an order of magnitude smaller [Budenstein, 1980; Frederickson et al., 1986].

Laboratory experiments confirm this breakdown threshold for a range of solids [Sørensen et al., 1999]. Note that these experiments also indicate that full breakdown still sometimes occurs at fields as low as $10^{6} \mathrm{~V} / \mathrm{m}$. This is because inhomogeneities within the dielectric can increase the local electric field by a factor of 10 with respect to the average electric field (see also work on the breakdown of dust by McDonald et al. [1980]). Inhomogeneities can include cavities, whether empty or gas filled, or even inclusions of material with different dielectric strength [Budenstein, 1980; Lisitsyn et al., 1998; Mazzanti et al., 2005]. Although inconclusive, additional vacuum experiments with lunar regolith simulant indicate that full breakdown may occur at $6 \times 10^{6} \mathrm{~V} / \mathrm{m}$ [Kirkici et al., 1996]. In Figures 7-9, the gray bar in the panel showing the electric field represents the range over which breakdown does not occur. All values lying outside that bar can induce breakdown. Therefore, the electric fields predicted in the previous section for large SEP events all exceed the threshold for dielectric breakdown in the lunar regolith within PSRs.

To create an electric field of these strengths requires a charged particle fluence (time-integrated flux) of $\sim 10^{10}-10^{11} \mathrm{~cm}^{-2}$ for spacecraft dielectrics [Garrett and Evans, 2001; Green and Dennison, 2008]. This fluence must occur on a timescale less than the dielectric's discharging timescale $\tau$. Otherwise, the dielectric will dissipate the deposited charge too quickly. As described above, the regolith's discharging timescale is about 20 days within PSRs. The above three SEP events all had fluences $>10^{10} \mathrm{~cm}^{-2}$ over the discharging timescale, thus meeting this criterion for dielectric breakdown.

Campins and Krider [1989] conducted a series of laboratory experiments exploring whether the high fluxes of electrons in Jupiter's radiation belts might stimulate dielectric breakdown on lo's surface. They bombarded natural mineral sulfur with 10-30 keV electrons. With fluxes similar to that in Jupiter's radiation belts $\left(10^{10}\right.$ electrons $\left.\mathrm{cm}^{-2} \mathrm{~s}^{-1}\right)$, breakdown did occur, even to the point of being visible in a darkened room. In their experiments, the charge was deposited in a layer in the upper $\sim 0.1 \mathrm{~mm}$ of sulfur.

Campins and Krider's experiments suggest that if dielectric breakdown occurs frequently, it could play an important role in the evolution of the surfaces of airless bodies. As they note, breakdown can melt and boil off material, in addition to causing mechanical, optical, and chemical changes (see also the review by Balmain [1987]). For example, breakdown can create craters on the surfaces of the dielectric [Bahder et al., 1982]. Furthermore, breakdown fragments dielectrics along the boundaries of inclusions where the dielectric constant changes. It has thus been proposed as a method for fragmenting rocks into their mineralogical components [Lisitsyn et al., 1998; Andres et al., 2001].

Our modeling of three large SEP events in the previous section indicates that dielectric breakdown may have occurred during those events within lunar PSRs. If this is the case, then we can roughly assess the importance of breakdown to the regolith by estimating the frequency of potentially breakdown-inducing SEP events. A comprehensive analysis by Feynman et al. [1993] of SEP events occurring from 1973 to 1991 found 89 events for which the daily averaged flux of $>1 \mathrm{MeV}$ protons was $>460 \mathrm{~cm}^{-2} \mathrm{~s}^{-1} \mathrm{sr}^{-1}$, their chosen flux threshold ( $1 \mathrm{MeV}$ was the lowest energy threshold in their study). About $20 \%$, or $\sim 18$ events, had fluences $>10^{10} \mathrm{~cm}^{-2}$ (see their Figure 3a). This corresponds to a frequency of $\sim 1$ event/yr potentially able to induce dielectric breakdown in the lunar regolith.

Two limitations to the results of Feynman et al. [1993] must be noted. First, the authors calculate fluence over a single SEP event. Since, however, the regolith's discharging timescale (20-40 days) is longer than typical events, the regolith may not have fully discharged before a subsequent event. Thus, both a single large event or a series of smaller events could induce breakdown. Future work is necessary to determine the importance of this. Second, the fluxes of lower energy SEP protons are typically higher than that of $>1 \mathrm{MeV}$ protons. For events with insufficient fluences of $>1 \mathrm{MeV}$ protons to instigate breakdown, the fluence of lower energy protons might still be sufficient to cause breakdown. Both these factors indicate that the breakdown-inducing SEP event rate is likely much higher than 1 events/yr.

Throughout the Moon's history, these potentially breakdown-inducing SEP events have affected more than just the present-day top $\sim 1 \mathrm{~mm}$ of regolith. Meteoritic impacts have continuously gardened the regolith, mixing it both vertically and horizontally [Arnold, 1975]. The area of an impactor's ejecta layer is much greater than the area excavated [Arnold, 1979]; therefore, gardening is primarily a protective process. Thus, 
most regolith affected by dielectric breakdown will typically be buried rather than destroyed or modified during excavation by an impact. Modeling by Arnold [1975] estimates that on average, any given layer of regolith will be buried with a protective $1 \mathrm{~mm}$ layer of regolith from another location after $1.2 \mathrm{Myr}$. Thus, each $1 \mathrm{~mm}$ layer within the regolith's gardened zone can experience breakdown-inducing SEP events for $1.2 \mathrm{Myr}-\mathrm{a}$ total of $\sim 1.2 \times 10^{6}$ breakdown-inducing events. This applies to all regolith within the gardened zone. (Note that as shown in Jordan et al. [2013], this exposure time to SEPs could be up to about twice as long, depending on the amount of gardening. For simplicity, however, we use the above exposure time.)

In light of the effects mentioned above, breakdown may play a significant role in the weathering of the regolith, especially within PSRs and polar areas with low average temperatures. Since breakdown preferentially occurs along the boundaries of changes in the dielectric [Lisitsyn et al., 1998; Andres et al., 2001], repeated breakdown would tend to fragment the regolith into its mineralogical components. Thus, such a large number of breakdown-inducing SEP events could cause the regolith within PSRs to have a reduced concentration of aggregates with respect to regolith in warmer regions.

This breakdown weathering could affect other airless bodies in the solar system. Mercury, for example, also has permanently shadowed regions with temperatures below $100 \mathrm{~K}$ [Vasavada et al., 1999]. The soil within those craters may therefore have a low conductivity. Furthermore, because Mercury is about $0.3 \mathrm{AU}$ from the Sun, SEP fluences are likely an order of magnitude greater there than at Earth. This means that PSRs on Mercury may experience more frequent dielectric breakdown than those on the Moon.

\section{Conclusions}

We have estimated for the first time the electric fields due to deep dielectric charging of the Moon by SEPs and GCRs. We have created a one-dimensional, two-layer, time-dependent model to calculate the lunar subsurface charging. Using CRaTER GCR data as an input, we find that GCRs create persistent electric fields up to $700 \mathrm{~V} / \mathrm{m}$. ACE/EPAM data during large SEP events show that such events create transient but strong fields that may even induce dielectric breakdown. We find peak electric fields to be at least on the order of $10^{7} \mathrm{~V} / \mathrm{m}$; the lower-energy SEPs that EPAM cannot detect may create much stronger fields. Also, the present layer of gardened regolith has likely experienced at least $1.2 \times 10^{6}$, and possibly many more, breakdown-inducing events. This high number of events likely reduces the percentage of aggregate particles in the regolith in PSRs by slowly fragmenting the particles along mineralogical boundaries during large SEP events.

There are several ways to test or adapt this first-order model. One test would require irradiating cryogenic regolith with proton and electron beams to determine whether the resulting charging and occurrence of dielectric breakdown match the model's predictions. The model could also be adapted, by incorporating a surface charging model, to predict the electric field above the Moon's surface, which may relate to the Lunar Prospector observations of surface charging during SEP events that were not readily explained by current balance at the surface [Halekas et al., 2009]. This model could also be applied to deep dielectric charging at other airless bodies in the solar system, such as Mercury, to estimate the importance of deep dielectric charging and breakdown weathering.

Acknowledgments

This work was supported by NASA grant NNG11PA03C and NNX10AB17A. The authors wish to thank Larry Townsend for helpful discussions. The authors also thank the ACE/EPAM team and its Principal Investigator Robert Gold of JHU/APL for providing the ACE data via CDAWeb at http://cdaweb. gsfc.nasa.gov/. LRO/CRaTER Level 2 data are available at the NASA Planetary Data System at http://pds.nasa. gov. The PSTAR and ESTAR results are available from the NIST website at http://www.nist.gov.

\section{References}

Andres, U., I. Timoshkin, and M. Soloviev (2001), Energy consumption and liberation of minerals in explosive electrical breakdown of ores, Miner. Process. Extractive Metall., 110(3), 149-157, doi:10.1179/mpm.2001.110.3.149.

Arnold, J. R. (1975), Monte Carlo simulation of turnover processes in the lunar regolith, Proc. Lunar Planet Sci., 6, $2375-2395$.

Arnold, J. R. (1979), Ice in the lunar polar regions, J. Geophys. Res., 84, 5659-5668, doi:10.1029/JB084iB10p05659.

Arnold, J. R., M. Honda, and D. Lal (1961), Record of cosmic-ray intensity in the meteorites, J. Geophys. Res., 66, 3519-3531, doi:10.1029/JZ066i010p03519.

Axford, W. I., E. Leer, and G. Skadron (1977), The acceleration of cosmic rays by shock waves, in International Cosmic Ray Conference, vol. 11, pp. 132-137, B'lgarska Akademiia na Naukite, Sofia.

Bahder, G., T. Garrity, M. Sosnowski, R. Eaton, and C. Katz (1982), Physical model of electric aging and breakdown of extruded polymeric insulated power cables, IEEE Trans. Power Apparatus Syst., PAS-101(6), 1379-1390.

Balmain, K. G. (1987), Arc propagation, emission and damage on spacecraft dielectrics: A review, J. Electrost., 20(1), 95-108.

Bamford, R. A., and et al. (2012), Minimagnetospheres above the lunar surface and the formation of lunar swirls, Phys. Rev. Lett., 081101 (8), doi:10.1103/PhysRevLett.109.081101.

Bedingfield, K. L., R. D. Leach, and M. B. Alexander (1996), Spacecraft system failures and anomalies attributed to the natural space environment, NASA Reference Publication 1390, Marshall Space Flight Center, Huntsville, Ala.

Bell, A. R. (1978), The acceleration of cosmic rays in shock fronts. I, Mon. Not. R. Astron. Soc., 182, 147-156. 
Berger, M. J., J. S. Coursey, M. A. Zucker, and J. Chang (2005), ESTAR, PSTAR, and ASTAR: Computer programs for calculating stopping-power and range tables for electrons, protons, and helium ions (version 1.2.3), National Institute of Standards and Technology, Gaithersburg, Md. [Avaialble at http://physics.nist.gov/Star, accessed 29 August 2012.]

Blandford, R. D., and J. P. Ostriker (1978), Particle acceleration by astrophysical shocks, Astrophys. J., 221, L29-L32, doi:10.1086/182658.

Bosqued, J. M., et al. (1996), Moon-solar wind interactions: First results from the WIND/3DP Experiment, Geophys. Res. Lett., 23, 1259-1262, doi:10.1029/96GL00303.

Budenstein, P. P. (1980), On the mechanism of dielectric breakdown of solids, IEEE Trans. Electr. Insul., El-15(3), 225-240.

Buhler, C., C. Calle, J. Clements, J. Mantovani, and M. Ritz (2007), Test method for in situ electrostatic characterization of lunar dust, in Aerospace Conference, pp. 1-19, IEEE, Big Sky, Mont., doi:10.1109/AERO.2007.352755.

Campins, H., and E. P. Krider (1989), Surface discharges on natural dielectrics in the solar system, Science, 245, 622-624, doi:10.1126/ science.245.4918.622.

Carrier, W. D. III, G. R. Olhoeft, and W. Mendell (1991), Physical properties of the lunar surface, in Lunar Sourcebook, edited by G. Heiken, D. Vaniman, and B. M. French, pp. 475-594, Cambridge Univ. Press, Cambridge.

Farrell, W. M., T. J. Stubbs, J. S. Halekas, R. M. Killen, G. T. Delory, M. R. Collier, and R. R. Vondrak (2010), Anticipated electrical environment within permanently shadowed lunar craters, J. Geophys. Res., 115, E03004, doi:10.1029/2009JE003464.

Feynman, J., G. Spitale, J. Wang, and S. Gabriel (1993), Interplanetary proton fluence model: JPL 1991, J. Geophys. Res., 98, 13,281-13,294, doi:10.1029/92JA02670.

Frederickson, A. R. (1977), Radiation induced currents and conductivity in dielectrics, IEEE Trans. Nucl. Sci., 24, 2532-2539, doi:10.1109/TNS.1977.4329251.

Frederickson, A. R. (1983), Electric discharge pulses in irradiated solid dielectrics in space, IEEE Trans. Electr. Insul., El-18(3), 337-349.

Frederickson, A. R., D. B. Cotts, J. A. Wall, and F. L. Bouquet (1986), Spacecraft Dielectric Material Properties and Spacecraft Charging, American Institute of Aeronautics and Astronautics, New York.

Frederickson, A. R., E. G. Holeman, and E. G. Mullen (1992), Characteristics of spontaneous electrical discharging of various insulators in space radiations, IEEE Trans. Nucl. Sci., 39, 1773-1782, doi:10.1109/23.211366.

Freeman, J. W., and M. Ibrahim (1975), Lunar electric fields, surface potential and associated plasma sheaths, Moon, 14, 103-114, doi:10.1007/BF00562976.

Garrett, H. B., and R. W. Evans (2001), Internal electrostatic discharge environment at Jupiter, in Spacecraft Charging Technology, vol. 476, edited by R. A. Harris, 609 pp. ESA Special Publication, Noordwijk, The Netherlands.

Gold, R. E., S. M. Krimigis, S. E. Hawkins III, D. K. Haggerty, D. A. Lohr, E. Fiore, T. P. Armstrong, G. Holland, and L. J. Lanzerotti (1998), Electron, proton, and alpha monitor on the advanced composition explorer spacecraft, Space Sci. Rev., 86, 541-562, doi:10.1023/A:1005088115759.

Gosling, J. T. (1993), The solar flare myth, J. Geophys. Res., 98, 18,937-18,950, doi:10.1029/93JA01896.

Green, N. W., and J. R. Dennison (2008), Deep dielectric charging of spacecraft polymers by energetic protons, IEEE Trans. Plasma Sci., 36, 2482-2490, doi:10.1109/TPS.2008.2003442.

Greenberg, P. S., D.-R. Chen, and S. A. Smith (2007), Aerosol measurements of the fine and ultrafine particle content of lunar regolith, Tech. Rep. NASA/TM-2007-214956, Glenn Research Center, Cleveland, Ohio.

Halekas, J. S., S. D. Bale, D. L. Mitchell, and R. P. Lin (2005), Electrons and magnetic fields in the lunar plasma wake, J. Geophys. Res., 110 , A07222, doi:10.1029/2004JA010991.

Halekas, J. S., G. T. Delory, D. A. Brain, R. P. Lin, M. O. Fillingim, C. O. Lee, R. A. Mewaldt, T. J. Stubbs, W. M. Farrell, and M. K. Hudson (2007), Extreme lunar surface charging during solar energetic particle events, Geophys. Res. Lett., 34, L02111, doi:10.1029/2006GL028517.

Halekas, J. S., G. T. Delory, R. P. Lin, T. J. Stubbs, and W. M. Farrell (2008), Lunar Prospector observations of the electrostatic potential of the lunar surface and its response to incident currents, J. Geophys. Res., 113, A09102, doi:10.1029/2008JA013194.

Halekas, J. S., G. T. Delory, R. P. Lin, T. J. Stubbs, and W. M. Farrell (2009), Lunar surface charging during solar energetic particle events: Measurement and prediction, J. Geophys. Res., 114, A05110, doi:10.1029/2009JA014113.

International Commission on Radiation Units (1993), Stopping Powers and Ranges for Protons and Alpha Particles, International Commission on Radiation Units and Measurements, Washington, D. C.

Jordan, A. P., T. J. Stubbs, C. J. Joyce, N. A. Schwadron, H. E. Spence, and J. K. Wilson (2013), The formation of molecular hydrogen from water ice in the lunar regolith by energetic charged particles, J. Geophys. Res. Planets, 118, 1257-1264, doi:10.1002/jgre.20095.

Kirkici, H., M. F. Rose, and T. Chaloupka (1996), Experimental study on simulated lunar soil: High voltage breakdown and electrical insulation characteristics, IEEE Trans. Dielectr. Electr. Insul., 3(1), 119-125.

Koons, H. C., J. E. Mazur, R. S. Selesnick, J. B. Blake, J. F. Fennell, J. L. Roeder, and P. C. Anderson (1998), The impact of the space environment on space systems, paper presented at 6th Spacecraft Charging Technology, 7-11, Hanscom AFB, Mass.

Krymskii, G. F. (1977), A regular mechanism for the acceleration of charged particles on the front of a shock wave, Akademiia Nauk SSSR Doklady, 234, 1306-1308.

Lisitsyn, I. V., H. Inoue, I. Nishizawa, S. Katsuki, and H. Akiyama (1998), Breakdown and destruction of heterogeneous solid dielectrics by high voltage pulses, J. Appl. Phys., 84(11), 6262-6267.

Mazzanti, G., G. C. Montanari, and L. A. Dissado (2005), Electrical aging and life models: The role of space charge, IEEE Trans. Dielectr. Electr. Insul., 12(5), 876-890.

McDonald, J. R., R. B. Mosley, and L. E. Sparks (1980), An approach for describing electrical characteristics of precipitated dust layers, J. Air Pollut. Control Assoc., 30(4), 372-376.

McGuire, R. E., and T. T. von Rosenvinge (1984), The energy spectra of solar energetic particles, Adv. Space Res., 4, 117-125, doi:10.1016/0273-1177(84)90301-6.

McKay, D. S., G. Heiken, A. Basu, G. Blanford, S. Simon, R. Reedy, B. M. French, and J. Papike (1991), The lunar regolith, in Lunar Sourcebook edited by G. Heiken, D. Vaniman, and B. M. French, pp. 285-356, Cambridge Univ. Press, Cambridge.

Ogilvie, K. W., J. T. Steinberg, R. J. Fitzenreiter, C. J. Owen, A. J. Lazarus, W. M. Farrell, and R. B. Torbert (1996), Observations of the lunar plasma wake from the WIND spacecraft on December 27, 1994, Geophys. Res. Lett., 23, 1255-1258, doi:10.1029/96GL01069.

Olhoeft, G. R., and D. W. Strangway (1975), Dielectric properties of the first 100 meters of the Moon, Earth Planet. Sci. Lett., 24, 394-404, doi:10.1016/0012-821X(75)90146-6.

Olhoeft, G. R., D. W. Strangway, and A. L. Frisillo (1973), Lunar sample electrical properties, in Proceedings of the Fourth Lunar Science Conference, vol. 4, pp. 3133-3149, Lunar and Planetary Institute, Houston, Tex.

Olhoeft, G. R., A. L. Frisillo, D. W. Strangway, and H. Sharpe (1974), Temperature dependence of electrical conductivity and lunar temperatures, The Moon, 9, 79-87, doi:10.1007/BF00565394. 
Paige, D. A., et al. (2010), Diviner lunar radiometer observations of cold traps in the Moon's South Polar Region, Science, 330, 479-482, doi:10.1126/science.1187726.

Papike, J., L. Taylor, and S. Simon (1991), Lunar minerals, in Lunar Sourcebook, edited by G. Heiken, D. Vaniman, and B. French, pp. 121-181, Cambridge Univ., Cambridge, U. K.

Pike, R. J. (1974), Depth/diameter relations of fresh lunar craters-Revision from spacecraft data, Geophys. Res. Lett., 1, 291-294, doi:10.1029/GL001i007p00291.

Reames, D. V. (2002), Magnetic topology of impulsive and gradual solar energetic particle events, Astrophys. J. Lett., 571, L63-L66, doi:10.1086/341149.

Robinson, P. A., Jr., and P. Coakley (1992), Spacecraft charging-Progress in the study of dielectrics and plasmas, IEEE Trans. Electr. Insul., 27, 944-960.

Smart, D. F., and M. A. Shea (1985), Galactic cosmic radiation and solar energetic particles, in Handbook of Geophysics and the Space Environment, edited by A. S. Jursa, pp. 6-1-6-29, Air Force Geophysics Laboratory, Bedford, Mass.

Sørensen, J., D. Rodgers, K. Ryden, P. Latham, G. Wrenn, L. Levy, and G. Panabiere (1999), Esa's tools for internal charging, in RADECS Fifth European Conference on Radiation and Its Effects on Components and Systems, pp. 27-33, IEEE, Fontevraud, doi:10.1109/RADECS.1999.858540.

Spence, H. E., et al. (2010), CRaTER: The cosmic ray telescope for the effects of radiation experiment on the Lunar Reconnaissance Orbiter Mission, Space Sci. Rev., 150, 243-284, doi:10.1007/s11214-009-9584-8.

Stubbs, T. J., J. S. Halekas, W. M. Farrell, and R. R. Vondrak (2007), Lunar Surface Charging: A Global Perspective Using Lunar Prospector Data.

Stubbs, T. J., W. M. Farrell, J. S. Halekas, J. K. Burchill, M. R. Collier, M. I. Zimmerman, R. R. Vondrak, G. T. Delory, and R. F. Pfaff (2014), Dependence of lunar surface charging on solar wind plasma conditions and solar irradiation, Planet. Space Sci., 90, 10-27, doi:10.1016/j.pss.2013.07.008.

Van Hollebeke, M. A. I., L. S. Ma Sung, and F. B. McDonald (1975), The variation of solar proton energy spectra and size distribution with heliolongitude, Sol. Phys., 41, 189-223, doi:10.1007/BF00152967.

Vaniman, D., R. Reedy, G. Heiken, G. Olhoeft, and W. Mendell (1991), The lunar environment, in Lunar Sourcebook, edited by G. Heiken, D. Vaniman, and B. French, pp. 27-60, Cambridge Univ., Cambridge, U. K.

Vasavada, A. R., D. A. Paige, and S. E. Wood (1999), Near-surface temperatures on Mercury and the Moon and the stability of polar ice deposits, Icarus, 141, 179-193, doi:10.1006/icar.1999.6175.

Violet, M. D., and A. R. Frederickson (1993), Spacecraft anomalies on the CRRES satellite correlated with the environment and insulator samples, IEEE Trans. Nucl. SCi., 40, 1512-1520, doi:10.1109/23.273511.

Walker, R. M. (1980), Nature of the fossil evdience: Moon and meteorites, in The Ancient Sun: Fossil Record in the Earth, Moon and Meteorites, edited by R. O. Pepin, J. A. Eddy, and R. B. Merrill, pp. 11-28, Pergamon Press, New York.

Whipple, E. C. (1981), Potentials of surfaces in space, Rep. Prog. Phys., 44, 1197-1250, doi:10.1088/0034-4885/44/11/002.

Willis, R. F., M. Anderegg, B. Feuerbacher, and B. Fitton (1973), Photoemission and secondary electron emission from lunar surface material, in Photon and Particle Interactions with Surfaces in Space, Astrophysics and Space Science Library, vol. 37, edited by R. F. Willis et al., pp. 389-401, Springer, Netherlands, doi:10.1007/978-94-010-2647-5_25.

Zimmerman, M. I., W. M. Farrell, T. J. Stubbs, J. S. Halekas, and T. L. Jackson (2011), Solar wind access to lunar polar craters: Feedback between surface charging and plasma expansion, Geophys. Res. Lett., 38, L19202, doi:10.1029/2011GL048880.

Zimmerman, M. I., T. L. Jackson, W. M. Farrell, and T. J. Stubbs (2012), Plasma wake simulations and object charging in a shadowed lunar crater during a solar storm, J. Geophys. Res., 117, E00K03, doi:10.1029/2012JE004094. 